\title{
PALINOLOGIA DE AMOSTRAS DA BACIA DE BONFIM, TERCIÁRIO DO ESTADO DE SÃO PAULO, BRASIL
}

\author{
MURILO RODOLFO DE LIMA' \\ RODOLFO DINO ${ }^{2}$
}

\section{RESUMO}

Embora frequientemente mencionada na literatura geológica. a Bacia de Bonfim (ocorrência também conhecida como "Linhito de Caçapava"), é, até os dias atuais, muito pouco conhecida do ponto de vista geológico. Entre os pontos de interesse que permanecem obscuros, destacam-se a idade e a gênese da sequência sedimentar.

O trabalho ora apresentado pretende preencher algumas das lacunas de informação existentes, através da análise palinológica de seus sedimentos. Três amostras representativas de diferentes litologias da bacia foram estudadas qualitativa e quantitativamente, fornecendo resultados interessantes. Cincoenta e sete espécies de palinomorfos e um escolecodonte foram identificados. A partir da aná. lise das associaçôes, uma idade eocênica pode ser estabelecida para os níveis portadores, depositados seguramente em condiçōes não marinhas. A idade atribuida, embora não permita conclusões definitivas a respeito da gênese da bacia, inviabiliza a correlação normalmente efetuada com sedimentos da parte superior da Formação Tremembé, unidade estratigráfica pertencente à vizinha Bacia de Taubaté.

\section{ABSTRACT}

Although frequently mentioned in geologic literature, the Bonfim Basin, commonly referred to simply as the "Caçapava Lignite". has been virtually unknown, until recently, from a geologic point of view. Among the interesting aspects still obscure are its age and origin.

The present paper attempts to fill some of the existing gaps in knowledge as a result of the qualitative and quantitative palynological analysis of three representative samples of the lithologies in the basin. Fifty-seven species of palynomorphs and one scolecodont were identified. The palynological associations establish an Eocene age for the strata and indicate deposition under fresh-water conditions. This age, although not permitting definitive conclusions as to the origin of the basin. invalidates the correlation usually made with sediments of the upper part of the Tremembe Forma. tion of the neighboring Taubaté Basin.

\section{INTRODUÇÃo}

Apesar de nāo apresentar grande extensāo superficial, principalmente quando comparada com outras bacias sedimentares do Estado de São Paulo, a Bacia de Bonfim tem sido, desde longo tempo, alvo de apreciável interesse, sobretudo nos aspectos que dizem respeito ao aproveitamento de seus jazimentos linhíticos. Mais conhecida na literatura especializada pelo tipo de sedimento que a caracteriza, ou seja, como o "Linhito de Caçapava", as publicaçőes a ela concernentes săo relativamente expressivas no que se refere a aspectos tecnológicos PAES LEME 1918, 1924; FELICISSIMO JR. 1940; BINIAMOVSKY 1943, etc). A ocorréncia chegou a ser considerada segundo FOURQU.

' Institu to de Geociencias, Universidade de Sāo Paulo.

2 Instituto de Pesquisas Tecnológicas (IPT).
REAN (1945, p. 185) "o depósito de linhito mais importante do Brasil, quer pela produção e método de trabalho". Apesar disto, muito pouco se conhece sobre os aspectos geológios da área, cuja exploraçåo econômica limita-se hoje ao rejeito da antiga mina. Vários pontos relacionados à sua gênese, evolução e idade permanecem obscuros. Um dos fatores que dificultam sobremaneira o esclarecimento dos problemas ligados à Bacia de Bonfim é a escassez de dados paleontológicos e estratigráficos.

O objetivo principal do presente trabalho é o de caracterizar, através de análises palinológicas, a idade e o ambiente em que esses sedimentos se depositaram, contribuindo assim para um melhor conhecimento da área em questão. 


\section{DADOS ESTRATIGRÁFICOS E PALEONTOLÓGICOS}

\section{Aspectos gerais}

Com um formato estreito e alongado. possuindo aproximadamente $219.000 \mathrm{~m}^{2}$, a Bacia de Bonfim acha-se situada na escarpa granítica da Serra do Mar, mais especifica- mente no seu contraforte (Serra do Jambeiro), a $12 \mathrm{~km}$ a sul da cidade de Caçapava (Fig. 1). Seu comprimento nฮ̃o ultrapassa os $1.100 \mathrm{~m}$, e a largura é de cerca de $200 \mathrm{~m}$. Possui orientação geral de direção W-SW-ENE e seus sedimentos apresentam um mergulho médio da ordem de 10 a $15^{\circ}$, podendo atingir valores de até $45^{\circ}$
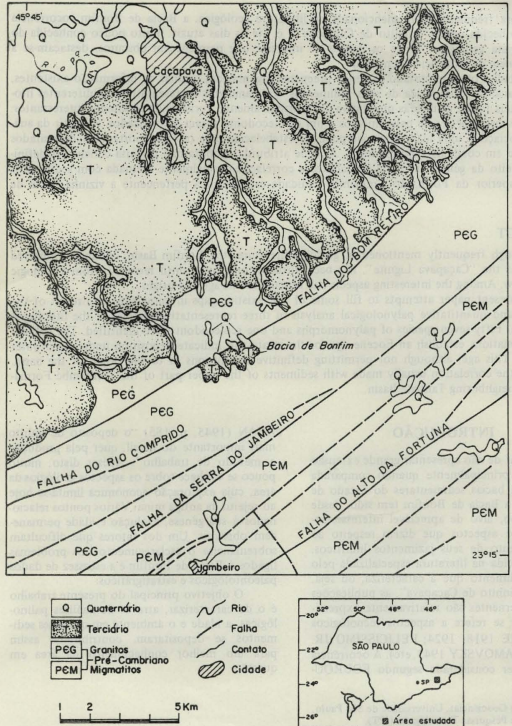

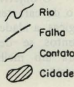

$5 \mathrm{Km}$

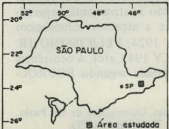

Fig. 1 -

Geologia e mapa de lo-

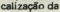
área ladap. tado de HASUI $e t$ al, 1978). 


\section{Litoestratigrafia}

Em função do grande interesse nos depósitos de linhito existentes na bacia (estimados em 1 milhão de toneladas), vários furos de sondagem já foram executados na área. BINIA. MOVSKY (1943) apresenta alguns dados referentes a 49 que foram efetuados pela então concessionária, a Cia. Carbonifera de Caçapava. Estes dados permitem concluir que o pacote sedimentar, irregular em função do tipo de embasamento, pode atingir valores da ordem de $80 \mathrm{~m}$. Este pacote nunca chegou a ser caracterizado formalmente em termos litoestratigráficos. Os furos de sondagem mencionados revelam um assoalho muito irregular (não atingido em vários deles), sobre o qual se depositaram os sedimentos da bacia, cuja seçāo esquemática é apresentada na Figura 2. A sequência inicia-se com a deposição de sedimentos arenosos, que gradativamente passam a sedimentos mais finos. Sobre essa camada arenosa assenta-

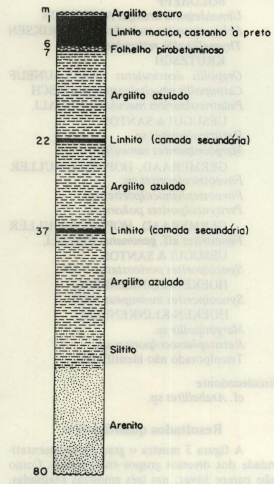

Fig. 2 - Perfil esquemático representativo de sequencia sedimentar da Bacia do Bonfim. se um argilito de coloraçato azulada, de espessura variável, contendo camadas secundárias de linhito. Acima desta sequência localiza-se uma camada de cerca de $1 \mathrm{~m}$ de espessura de folhelho pirobetuminoso, sobre o qual repousa a camada principal de linhito. Esta, de natureza lenticular, alcança em alguns pontos $5 \mathrm{~m}$ de espessura, possuindo coloraçao pardacenta. Finalmente, capeando a camada linhítica, verificase outra camada de argilito, escura, que passa gradualmente a solo.

\section{Paleontologia}

A exemplo dos dados estratigráficos, as informaçōes paleontológicas são também escassas, além de serem tratadas de modo bastante superficial.

O primeiro registro de fósseis deve-se a Lögren (in PAES LEME, 1918). Foram mencionados abundantes restos vegetais na camada de linhito, incluindo coniferas (Podocarpus lamberti) além de vária Rutales (incluindo o gènero Zanthoxylum) e Ebenales. Outras citaçбes såo encontradas em OLIVEIRA (1937) e OLIVEIRA \& LEONARDOS (1978) indicando a ocorrência de folhas de gramíneas e sementes arredondadas no linhito, bem como troncos e ramos linhitificados na camada argilosa.

Além destas mençōes, nenhuma informação, estudo ou coleta de fósseis foi, até o momento, realizada na área.

\section{DADOS PALINOLÓGICOS}

\section{Material e métodos}

O material analisado é proveniente do rejeito da jazida em exploração existente na bacia. Foram coletadas três amostras, a primeira correspondendo ao folhelho pirobetuminoso, a segunda ao linhito e a terceira ao argilito situado abaixo do nivel de folhelho. As amostras foram previamente preparadas de acordo com os procedimentos palinológicos usuais, ou seja, com utilização de $\mathrm{HCl}$, $\mathrm{HF}$ e $\mathrm{ZnCl}_{2}$ para eliminação dos componentes inorgânicos. Após este tratamento os resíduos foram oxidados com $\mathrm{HNO}_{3}$. Foram montadas 3 lâminas de cada amostra, que após a análise foram depositadas na coleção científica do DPE-IG/USP sob os n9s GP/4T-114 a 122 . A análise foi efetuada em fotomicroscópio Zeiss pertencente ao DPE-IG/USP.

\section{Resultados qualitativos}

Cincoenta e sete espécies de palinomorfos e um escolecodonte foram identificados, tendo sido para isto consultados, entre outros, os tra- 
balhos de ARCHANGELSKY (1973), CHATEAUNEUF (1980), FREYLE (1972), GERMERAAD et al, (1968), GONZALEZ-GUZMAN (1967), JAN DU CHENE et al (1978), LEIDELMEYER (1966), LIMA e AMADOR (no prelo), LIMA \& SALARD-CHEBOLDAEFF (1981), LIMA et al (no prelo), REGALI et al. (1974), SAXENA (1979), SALARD-CHEBOL. DAEFF (1978), VAN DER HAMMEN \&WIJMSTRA (1964) e VAN HOEKEN-KLINKENBERG (1966).

A lista completa é a seguinte:

Algas

Pseudoschizea circula

(WOLFF) CRISTOPHER

Briófitas

Stereisporites (Distzonosporis) sp.

Stereisporites (Duplozonosporis) sp.

Pteridófitas

Todisporites sp.

Biretisporites crassilabratus ARCHANGENLSKY

Leiotriletes sp. in FREYLE, 1972, p. 43. est. 1, fig. 1.

Divisisporites enormis PFLUG

Undulatisporites pseudobrasiliensis PFLUG

Hamulatisporis sp.

Cicatricosisporites dorogensis POTONIE \& GELLETICH

Lycopodiumsporites austroclavatidites (COOKSON) POTONIE

Gleicheniidites apilobatus BRENNER

Gleicheniidites (Toridistalisporis) toriconcarus KRUTZSCH

Gleicheniidites (Radiatisporis) radiatus (BOLKHOVITINA) KRUTZSCH

Gleicheniidites (Triremisporites) sp. of. $G$. $(T$.) delcourtii DÖRING

Gleicheniites sp.

Laevigatosporites ovatus WILSON \& WEBSTER

Laevigatosporites sp.

Polypodiisporites ornatus SAH

Verrucatosporites tennelis (KRUTZSCH)

Gimnospermas

Pityosporites sp. cf. $P$. elongatus NAKOMAN

Pityosporites microalatus (POTONIE) THOMSON \& PFLUG

Podocarpidites microreticuloidata COOKSON

Podocarpidites sp.

Ephedripites (Distachyapites) fusiformis (SHAKMUNDES) KRUTZSCH

Cycadopites giganteus STANLEY

Cycadopites sp.
Angiospermas

Arecipites sp.

Papillamonocolpites sp.

Platoniapollenites sp. in SAXENA, 1979. p. 135 , est. 2 , fig. 36 .

Retitricolpites golii DUENAS

Retitricolpites clarensis GONZALEZ GUZMAN

Retitricolpites geranioides (COUPER) BRENNER

Retitricolpites sp.

Retitrescolpites sp.

Verrustephanocolpites sp.

Propylipollis concretus (HARRIS) MARTIN \& HARRIS

Scabratriporites simpliformis VAN HOEKEN-KLINKENBERG

Proteacidites dehaani GERMERAAD, HOPING \& MULLER

Proteacidites sp. cf. P. rectomarginatus COOKSON

Corsinipollenites undulatus (GONZALEZ GUZMAN) LIMA \& SALARD-CHE. BOLDAEFF

Ulmoideipites krempii ANDERSON

Echiperiporites tschudyi FREDERIKSEN

Thymellipollis retisculpturius KRUTZSCH

Orapollis denticulatus CHATEAUNEUF Catinipollis geiseltalensis KRUTZSCH

Psilatricolporites maculosus REGALI, UESUGUI \& SANTOS

Retitricolporites sp.

Margocolporites vanwïhei GERMERAAD, HOPING \& MULLER

Foveotricolporites sp.

Foveostephanocolporites sp.

Perisyncolporites pokornyi GERMERAAD, HOPING \& MULLER

Fenestrites aff. gemmatus REGALI, UESUGUI \& SANTOS

Syncolporites poricostatus VAN HOEKEN-KLINKENBERG

Syncolporites incomptus VAN HOEKEN-KLINKENBERG

Marginipollis sp.

Retistephanocolporites sp.

Tricolporado não identificado

Escolecodonte

cf. Arabellites sp.

\section{Resultados quantitativos}

A figura 3 mostra o grau de representatividade dos diversos grupos encontrados. Como não parece haver, nas trés amostras analisadas, nenhuma diferença cronológica, as variaçōes verificadas na composição microflorística de- 
vem ser atribuidas a fatores ambientais, e, eventualmente, de preservação. $\mathrm{Da}$ análise dos gráficos, as seguintes observações podem ser efetuadas:

Nas três amostras estudadas, a quantidade de esporos (somatório dos grupos triletes e monoletes) é marcante, oscilando entre 40 e $60 \%$. Tal comportamento mostra com clareza a grande influência de vegetação ar-

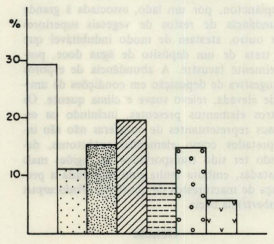

Folheilho

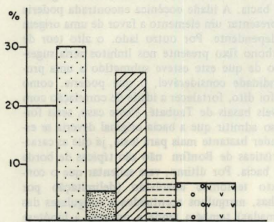

Argilito
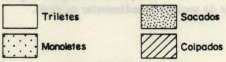

bustiva ou herbácea, preferencialmente palustre, na composiçāo do espectro polínico. Estes dados estariam mais em acordo com um pequeno corpo aquoso, ou uma reentrância bem protegida de um corpo aquoso de maiores dimensōes. Vale ainda a pena mencionar que eles incluem nāo só pteridófitas mas também algas e briófitas, o que tende a corroborar a observação efetuada.

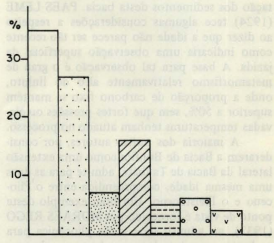

Linhito

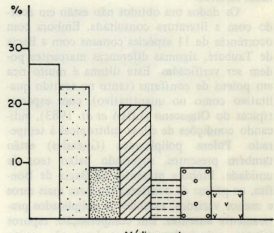

Médio geral

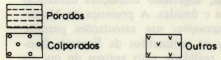

Fig. 3 - Representaçăo quantitativa dos principais grupos de palinomortos encontrados.

Polens de coniferas, apesar de presentes, sáo relativamente pouco expressivos, variando em percentagem entre 5 e $15 \%$. São, além disto, pouco diversificados, destacando-se o gênero Podocarpidites pela maior expressão. Deve ser ressaltado que estes polens, pela alta capacidade de transporte, representam não apenas a vegetação local, mas sim de toda a regiẩo circunvizinha.

Polens colpados são muito bem represen- 
tados (variando entre 18 e $25 \%$ ) em comparação com porados e colporados, refletindo uma relativa abundância de monocotiledôneas, especialmente de palmeiras, nas vizinhanças do depósito.

\section{DISCUSSÃO E CONCLUSÕES}

\section{Idade}

Muito pouco se tem falado acerca da datação dos sedimentos desta bacia. PAES LEME (1924) tece algumas consideraçōes a respeito ao dizer que a idade não parece ser tão recente como indicaria uma observação superficial da jazida. A base para tal observação é o grau de metamorfismo relativamente alto do linhito, onde a proporção de carbono fixo se mantém superior a $50 \%$, sem que fortes pressōes ou elevadas temperaturas tenham atuado no processo.

A maioria dos outros autores, por considerarem a Bacia de Bonfim como uma extensão lateral da Bacia de Taubaté, admite para as duas uma mesma idade, compreendida entre o Plioceno e o Pleistoceno. Um bom exemplo deste ponto de vista é fornecido por MORAES REGO (1933), ao admitir uma idade pliocênica para as bacias mencionadas, com base em observaçōes sobre a sequêtncia de fenômenos epirogê. nicos inferida.

Os dados ora obtidos não estão em acordo com a literatura consultada. Embora com ocorrência de 11 espécies comuns com a Bacia de Taubaté, algumas diferenças marcantes podem ser verificadas. Esta última é muito rica em polens de coníferas (tanto no sentido qualitativo como no quantitativo), com espécies típicas do Oligoceno (LIMA et al. 1983), indicando condiçōes de clima subtropical à temperado. Polens poliplicados (Gnetales) estāo também presentes, sugerindo baixo teor de umidade. Embora presentes na Bacia de Bonfim, os polens de conifferas são bem mais raros e menos variados, estando os poliplicados praticamente ausentes. Em compensação, esporos triletes, principalmente monoletes são muito mais comuns, sugerindo condiçōes climáticas mais quentes e úmidas. A presença de Margocolporites vanwijhei nas associaçōes permite concluir que os sedimentos da Fazenda Bonfim sāo em realidade mais antigos do que os de Taubaté, já que esta espécie, no Brasil, caracteriza o Eoceno.

Assim, a idade do "Linhito de Caçapava" seria mais antiga que a parte conhecida da Formação Tremembé mas poderia perfeitamente ter o seu equivalente cronológico em níveis mais profundos da Bacia de Taubaté, ainda nāo datados. Isto é possível porque os dados palinológicos desta última referem-se unicamente aos 100 metros mais superiores da Formação Tremembé (LIMA et al 1983). unidade também possuidora de folhelhos betuminosos (com espessura total estimada em cerca de $400 \mathrm{~m}$ ).

\section{Ambiente}

A ausência absoluta de elementos do microplâncton, por um lado, associada à grande abundância de restos de vegetais superiores por outro, atestam de modo indubitável que se trata de um depósito de água doce, possivelmente lacustre. A abundância de esporos é sugestiva de deposição em condições de umidade elevada, relevo suave e clima quente. Os outros elementos presentes, incluindo os es. cassos representantes de coníferas nāo sāo interpretados como elementos autóctones, devendo ter sido transportados de regiōes mais afastadas, embora tenha sido registrada a presença de macrorestos de coniferas (Podocarpus lamberti) na bacia.

\section{Gênese}

Os dados obtidos até o momento não permitem reconstruir de modo direto a gênese da bacia. A idade eocênica encontrada poderia representar um elemento a favor de uma origem independente. Por outro lado, o alto teor de carbono fixo presente nos linhitos seria sugestivo de que este esteve' submetido a uma profundidade considerável, o que poderia, como já foi dito, fortalecer a idade de correlação com níveis basais de Taubaté. Neste caso, seria forçoso admitir que a bacia original deveria se estender bastante mais para leste, já que as características de Bonfim nao são típicas de borda de bacia. Por último, vale salientar que o contexto tectônico envolvido (delimitação por falhas, mergulhos acentuados e irregulares das camadas) também tende a reforçar a hipótese de ter havido um soerguimento considerável nesta área, com consequiènte remoçđo de grande parte da seqüência sedimentar original.

\section{AGRADECIMENTOS}

Os autores agradecem ao Prof. Dr. T. R. Fairchild pela revisão do abstract, bem como ao Prof. Dr. A. C. Rocha Campos pelo convite para a apresentação deste trabalho na sessão regular da Academia Brasileira de Ciências de $8 / 11 / 83$. 


\section{BIBLIOGRAFIA}

ARCHANGELSKY, S. - 1973 - Palinologia del Paleoceno de Chubut. I. Descripciones sistemáticas, Ameghiniana 10(4): 339-399.

BINIAMOVKSY, O. - 1943 - Nota sobre o estado atual de exploraçāo de linhito em Caçapava, O IGG(1)2: 208-209

CHATEAUNEUF, J. J. - 1980 - Palynostratigraphic el Paleoclimatologie de I'Eocene Supérieur er de IOligocene du Bassin de Paris (France), Mém, du BRGM 116, 357 p.

FELICISSIMO JR., J. - 1940 - Linhito e Turfa. Bol. IGG 27: 273-274.

FOURQUREAN, W.E. - 1945 - Linhito de Caçapava, Estado de Säo Paulo. Bol. DNPM(DFM) 74: $185-198$.

FREYLE, C. - 1972 - Estudio palinológico de la Formation Cerro Dorotea (Maestrichtiano-Paleoceno) de la Provinciz de Santa Cruz. I. Rev. Mus. de la Plata (n.s.) Paleont. 6: 39-63.

GERMERAAD, C.A.; HOPPING, C.A.; MULLER, J. - 1968 - Palynology of Tertiary sediments from tropical areas. Rev. Palaeobot. Paly nol. 8(3): 189-348.

GONZALEZ-GUZMAN, A.E. - 1967 - A palynological study on the Upper Los Cuenos and Mirador Formations. Ed. Brill (Leyden), 68 p.

HASUI, Y.; PONCANO, W.L,; BISTRICHI, C.A; STEIN, D.P.; GALVÃO, C.A.C.F.; GIMENEZ, A.F.; ALMEIDA, M.A.; PIRES NETO, A.G.; MELO, M.S.: SANTOS, M.C.R - 1978 - Geologia da regiäo administrativa 3 (Vale do Paraiba) e parte da regiäo administrativa 2 (Litoral) do Estado de São Paulo. Monogr. IPT 1: 1.78.

INSTITUTO DE PESQUISAS TECNOLOGICAS (IPT) - 1978 - Pesquisa de Turfa e Linhito no Vale do Paraíba. ReL. Int. IPT/CESP no $11.572,59 \mathrm{p}$.

JAN DU CHENE, R, E.; ONYIKE, M. S.; SOWUNMI, M. A. - 1978 - Some new Eocene pollen of the Ogwashi-A sa. ba Formation, Southeastern Nigeria. Rev. Esp. Micropal. 10(2): 285-322.

LEIDELMEYER, P. - 1966 - The Paleocene and Lower Eocene pollen-flora of Guyana. Leidse Geol. Meded. 38 : 49-70.

LIMA, M.R. de \& AMADOR, E.S. - 1983 - Análise palinológica de sedimentos da Formacão Resende, Terciário do Estado do Rio de Janeiro, Brasil. Anais do $8^{\circ}$ Congr. Bras. Paleontol., Rio de Janeiro (no prelo).

LIMA, M.R. de \& SALARD-CHEBOLDAEFF, M. - 1981 - Palynologie des Bassins de Gandarela et Fonseca (Eocene de l'État de Minas Gerais, Brésil). Bol. IG 12:33-54.

LIMA, M.R, de; SALARD-CHEBOLDAEFF, M.; SUGUIO, K. - 1983 - Etude palynologique de la Formation Tremembé, Tertiaire du Bassin de Taubaté (État de Säo Paulo, Brésill), d'après les èchantillons du sondage n.o 42 du CNP. A nais do 89 Congr. Bras. Paleontol., Rio de Janeiro (no prelo).

MORAES REGO, L.F. de - 1933 - As formaçóes cenozdicas de Săo Paulo. An. Esc. Politecn., Sâo Paulo, 2 : 235-247.

OLIVEIRA, A.I. - 1937 - Lenhito no Brasil. Miner. Metal. 2(8): 105-107.

OLIVEIRA, A.I. \& LEONARDOS, O.H. - 1978 - Geologia do Brasil, 3. Ed. Col. Mossoroense 72:685-686.

PAES LEME, A. B. - 1918 - Sobre a formaçfo de linhito de Capapava, 37 p.. est, Pap. Macedo, Rio de Janeiro.

PAES LEME, A.B. - 1924 - A génese do lenhito do norte de Säo Paulo. Bol. Serv. Geol. Mineral. do Brasil. 7 : 51-54.

REGALI, M.S.P.; UESUGUI, N.; SANTOS, A.S. - 1974 - Palinologia dos sedimentos meso-cenozóicos do Brasil. II. Bol. Tecn. Petrobrás 17(4): 263-301.

SALARD-CHEBOLDAEFF, M. - 1978 - Sur la palynoflore maestrichtienne et tertiaire du Bassin Sedimentaire Littoral du Cameroun. Pollen et Spores 20(2):215-260.

SAXENA, R.K. - 1979 - Palynology of the Matanomadh Formation in type area, Northwestern Kutch, India (Part. 2). The Palaeobotanist 26(2): 130-143.

VAN DER HAMMEN, T. \& WIJMSTRA, T. - 1964 - A palynological study on the Tertiary and Upper Cretaceous of British Guiand. Leidse Geol. Meded. 30:183-241.

VAN HOEKEN-KLINKENBERG, P.M.J. - 1966 - Maestrichtian. Paleocene and Eocene pollen and spores from Nigeria. Leidse Geol. Meded. $38: 37-48$. 


\section{LEGENDA DAS ESTAMPAS (Todas as figuras aumentadas $800 \mathrm{X}$}

\section{Estampa I}

Fig. 1. Todisporites sp. Läm. GP/4T-119. Fig. 2. Stereisporites (Distzonosporis) sp, Làm. GP/4T-1 16, Fig. 3. Stereisporites (Duplozanosporis) sp. Lâm. GP/4T-116. Fig. 4. Biretisporites crassilabratus. Lám, GP/4T-120, Fig. 5 . Leiotriletes sp. Lâm. GP/4T-121. Fig. 6. Divisisporites enormis. Lảm. GP/4T-121. Fig. 7. Gleicheniidites apilobatus. Lâm, GP/4T-116. Fig. 8. Undulatisporites pseudobrasiliensis Làm, GP/4T-116. Fig. 9-10. Hamulatisporis sp. Làm. GP/4T-1 16. Fig. 11. Cicatricosisporites dorogensis, Làm, GP/4T-117. Fig. 12. Cicatricosisporites dorogensis. Làm. GP/4T-121, Fig. 13. Cicatricosisporites dorogensis, Lâm. GP/4T-117. Fig. 14, Lycopodiumsporites austroclavatidites, Làm, GP/4T-117. Fig. 15. Gleicheniidites (Toridistalisporis) toriconcavus, Läm, GP/4T-116, Fig, 16, Gleicheniidites (Radiatisporis) radiatus, Lam, GP/4T-121.

\section{Estampa II}

Fig. 1 - Gleicheniidites (Triremisporis) sp, cf, G. (T,) delcourtii, Lâm. GP/4T-115. Fig. 2. Gieicheniites sp. Låm. GP/4T-119. Fig. 3. Laevigatosporites ovatus, Lâm, GP/4T-119. Fig. 4. Laevigatosporites sp. Lảm. GP/4T-119. Fig. 5. Polypodissoorites ornatus, Lâm, GP/4T-114. Fig, 6. Verrucatosporites tennelis. Lâm, GP/4T-121. Fig, 7. Pityosporites sp. cf. P. elongatus. Lam. GP/4T-118. Fig. 8. Podocarpidites microroticu/oidata. Lam. GP/4T-119. Fig. 9. Podocaroidites sp. Lâm. GP/4T-119. Fig. 10. Pityosporites microalatus. Làm. GP/4T-115. Fig. 11. Ephedripites (Distachyapites) fusiformis. Lảm. GP/4T-115. Fig. 12. Cycadopites giganteus. Lâm. GP/4T-115. Fig. 13. Cycadopites sp. Làm, GP/4T-115. Fig. 14, Arecipites sp. Làm, GP/4T-116. Fig, 15, Papillamonocolpites sp. Lâm. GP/4T-115. Fig.16. Retitricolpites geranioides. Låm. GP/4T-117. Fig. 17. Retitrescolpites sp. Lâm. GP/4T-119. Fig. 18, Retitricolpites goliii. Lâm. GP/4T-119. Fig. 19. Platoniapollenites sp. Lâm. GP/4T-122. Fig. 20. Retitricolpites clarensis. Làm. GP/4T. 118. Fig. 21. Retitricolpites sp. Lâm. GP/4T-1 19.

\section{Estampa III}

Fig. 1. Verrustephanocolpites sp. Läm. GP/4T-115. Fig. 2. Propylipollis concretus, Lảm, GP/4T-117. Fig. 3. Scabratriporites simpliformis. Lam. GP/4T-119. Fig. 4. Protescidites sp. cf. $P$. rectomarginatus. Lám. GP/4T-116. Fig. 5. Proteacidites dehasni. Lâm. GP/4T-117. Fig. 6. Corsinipollenites undulatus. Lâm. GP/4T-122. Fig. 7. Uimoideipites krempii. Lâm. GP/4T-117. Fig. 8. Echiperiporites tschudyi. Lâm. GP/4T-117. Fig. 9. Thymellipoillis retiscu/pturius. Làm, GP/4T-114. Fig, 10. Orapollis denticulatus. Lảm, GP/4T-122. Fig, 11, Catinipollis geise/talensis, Lâm. GP/4T-1 18. Fig. 12. Retitricolporites sp. Lam. GP/4T-121. Fig. 13. Psilatricolporites maculosus. Lâm. GP/4T-122. Fig. 14. Foveotricolporites sp. Lăm. GP/4T-117. Fig. 15. Margocolporites vanwijhthei. Läm. GP/4T-118. Fig. 16. Perisyncolporites pokornyi. Lầm. GP/4T-116. Fig. 17. Perisyncolporites pokornyi. Lâm. GP/4T-114. Fig. 18. Fe. nestrites aff. gemmatus. Läm. GP/4T-118. Fig. 19. Tricoiporado năo identificado. Lam. GP/4T-115. Fig. 20. Synco/. porites poricostatus. Làm. GP/4T-114. Fig. 21. Syncolporites incomptus. Lâm. GP/4T-117. Fig. 22. Marginipollis sp. Làm. GP/4T-120. Fig. 23. Retistephanocolporites sp. Làm, GP/4T-114, Fig. 24, Pseudoschizea circula. Lâm, GP/ 4T-122. Fig. 25. cf. Arabellites sp. Lâm, GP/4T-116. 

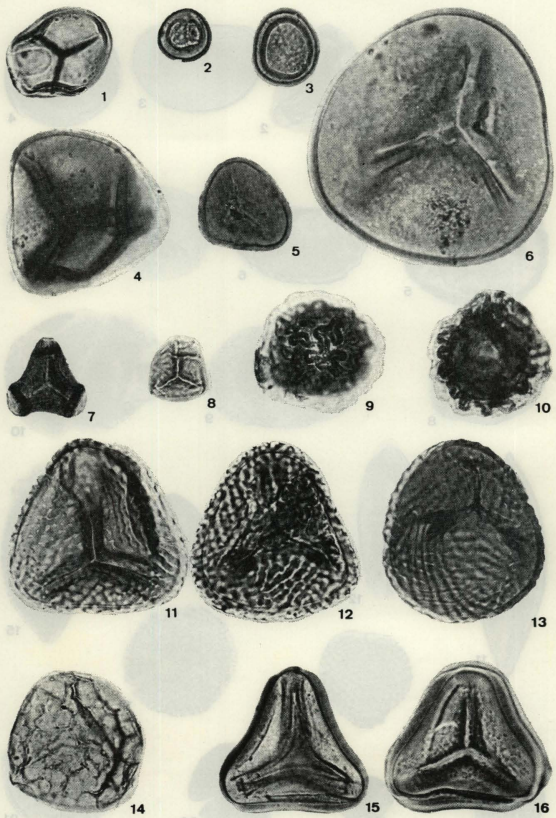

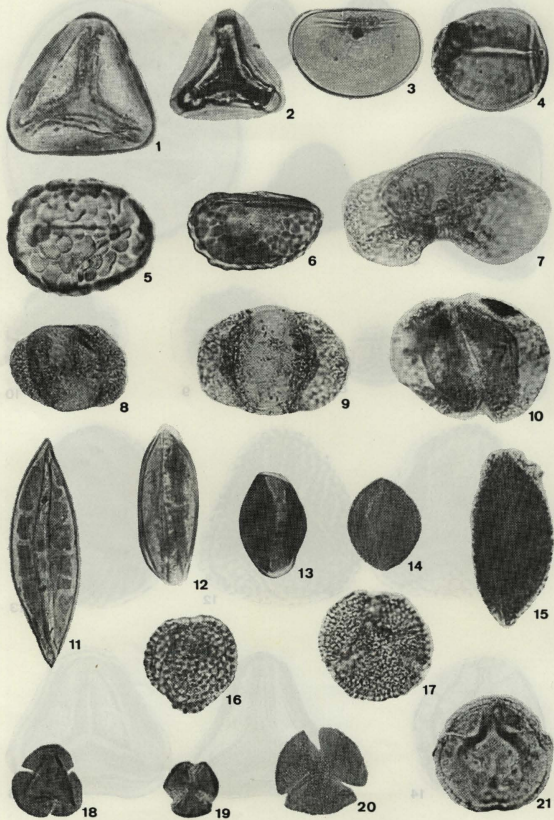

12
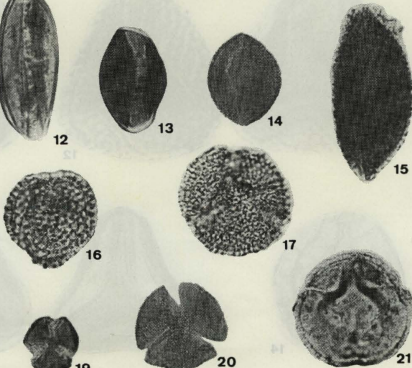

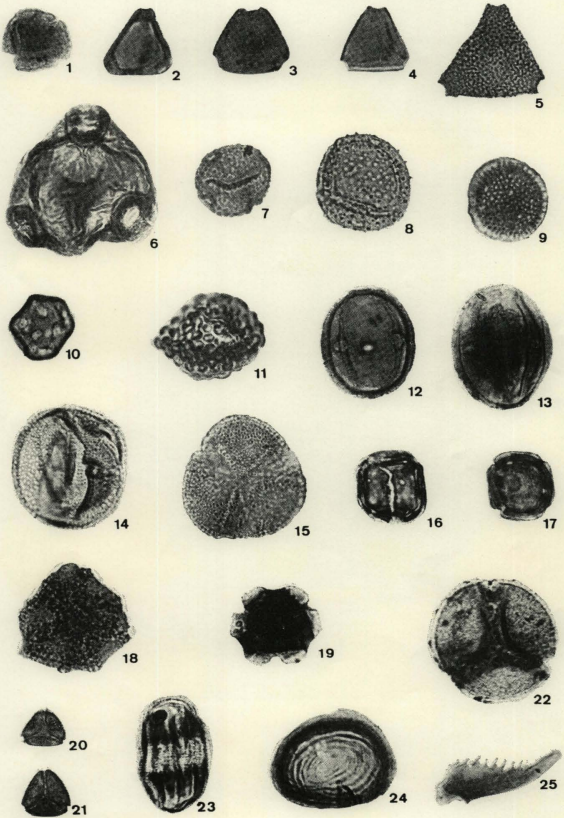